\title{
A Web-Based Aggregate Information Portal
}

\author{
Donatus I. Bayem, Henry O. Osuagwu, Chimezie F. Ugwu
}

\begin{abstract}
A Web portal aggregates an array of information for a target audience and affords a variety of services including search engines, directories, news, e-mail, and chat rooms, and they have evolved to provide a customized gateway to Web information. Also, a high level of personalization and customization has been possible. The portal concept could further be established to function as a classy Web interface that can serves as sustenance for variety of the task performance. The aggregate information Web portal will serve as portals for the information needs of users on the web. The Web based portal enable marketing of users broadly across a wide variety of interests. Most of the popular usage of the Web based aggregate information portal probably refers to the visual and user interface (UI) design of a Web site. It is a crucial aspect since the visitor is often more impressed with looks of website and easy to use rather than about the technologies and techniques that are used behind the scenes, or the operating system that runs on the web server. In other words, it just does not matter what technologies that is involved in creating, when the site is hard to use and easy to forget. This paper explores the factors that must be painstaking during the design and development of a Web based aggregate information portal. Design as a word in the context of a Web application can mean many things. A working Web based aggregate information portal, kaseremulticoncept was developed to support the various users' task performances. A number of technologies was studied and implemented in this research, which includes multi-tier architecture, server and client side scripting techniques and technologies such as PHP programming languages and relational databases such as MySQL, Structured Query language (SQL) and XAMPP Server.
\end{abstract}

Index Terms - Web portal, Aggregate, Information portal, kaseremulticoncept.

\section{INTRODUCTION}

Data mining on the internet has increased since the introduction of the portal concept to the Web, Web portals abruptly turn out to be the most promising Web services. Mining and integrating data from various Web sites have become easier with the arrival of powerful Web data extraction tools. This development has set in motion a phenomenon termed web aggregation [1] which is a procedure of bringing together information from various Web sources. The information collection from a mixture of cooperating and non-cooperating sources may consist of images, Web pages, and other types of files, has bring about countless number of Web aggregators.

In the recent time, most large organization employ portals as component of their working procedure. As more Web portals

Submitted on April 21, 2021

Published on May 12, 2021.

Donatus I. Bayem, Nigeria Maritime University, Delta State, Nigeria (e-mail: dbayem ${ }^{\circledR}$ gmail.com)

Henry O. Osuagwu, Nigeria Maritime University, Delta State, Nigeria.

(corresponding e-mail: henry.osuagwu.pg68120@unn.edu.edu) became accessible, portals now possess the capacity to incorporate different information sources and allow easier entrance to existing application within an organization [2]. The idea about a Portal was data accumulation from diverse sources and making of a solitary purpose of access to data a library of classified and customized content [3]. Rather than spending a lot of time searching through numerous locations, portals aggregate the relevant information at a single, easyto-remember location. The portal capability to aggregate data between stationary content blocks and Web application result in saving resources like time and money [4].

Establishing a Web based Information Aggregator Portal has certain useful module of arranging data by Real Simple Syndication (RSS) channels from diverse Internet sources, on the motivation behind the aggregator. However, most aggregator sites have made these practice to be lucrative by charging for adverts on their aggregator pages or turning into an offshoot of the Internet shopping destinations syndicating information from it. The arrival of the aggregator system has helped the internet users worldwide to provide a significance added e-services, by gathering relevant data on the Web and turns them into useful information [5]

An online data entrances have progressed to giving a tweaked door to Web data [6,7]. In an online data entrance, it is conceivable to have an abnormal state of personalization and customization. The gateway idea could further be assembled to work with the arrangement that will permit development of an effective online networking listening post, gratis. A Web entryway is the client interface, an entrance way to a specific site. When we get on the Internet and visit a site (for occurrence an organization or association), we utilize the Web entry to get to particular ranges of the site in light of our client consents. A key element of an online total data entry is to convey organized data to Web programs group of onlookers. It is a site that unites the client to different sites [8].

Designing and executing a Web based Information Aggregators Portal will assume cogently critical part in news transport for online gatherings of people. The goal of this research is to design an easy-to-use portal for aggregate information in some particular area of interest. We enhanced the news aggregate information to provide a single source for each headline and do not cluster together news articles from different sources reporting on the same story. This results in a simple laundry list of different top stories, each with a unique single source for content.

The rest of this paper will be structured in the following order: Section II presents the related works. In section III, Web-based portal design and development process was

Chimezie F. Ugwu, Institute of Management and Technology, Enugu, Nigeria.

(e-mail: chimeziefedricku ${ }^{@}$ gmail.com). 
presented. System implementation and performance evaluation was presented in section IV. Concluding remarks and limitations of the proposed Web base Information Aggregators Portals are discussed in section V and VI, respectively.

\section{RELATED WORKS}

The writing audit concentrate on taking a top to bottom take a glimpse at an electronic total data gateway. The structure of an online entry is thus: its sorts, substance, capacities, appearance, and nature. Ovum as examiner and counseling organization [9] has set up a point by point scientific classification of Portal sorts, parts, and their various leveled affiliations included. An up-and-running entryway does not just comprise of either a decent joint effort support or a decent incorporation of the data sources rather it includes a very much coordinated blend of the rudimentary gateway functionalities. Georgios Palioras et al. [10] optimized the previous version of Personalized News Service (PNS) by incorporating into the system an improved way of various services available by the Personalization Server (PServer) and presents an additional personalization solution. Several additional news sources were incorporated, and the user's interface has increased appreciably, which was noted as a major constraint of the users in the evaluation of the aforementioned system.

The work in [5] utilized the User Centered Design (UCD) technique to develop the aggregator system for an online journal. The UCD technique exploited in an online journal aggregator system, takes the idea of a Web portal to build an interface system that connect researchers, journal, journal managers and visitor. Measurements were taken using Linkert method to ascertain the degree of acceptance and support of potential users of the aggregator journal system. The practicality and interface of the online journal aggregator system have met the usefulness, with users' acceptance favorite for three attributes of the system, that is: content aspect, accessibility and direction finding.

Fatimah Ali et al. [11] develop an easy to use web-based educational platform for children with special needs from the design step to the implementation. The proposed scheme was developed using the Unified Modeling Language (UML) for the design, while ASP.NET, Hypertext Markup Language (HTML), Cascading Style Sheet (CSS) were utilized as programming language. The proposed scheme covers basic explanations of what is learned in selected school syllabus such as letters, numbers, and mathematical operations where it contains worksheets and extracurricular activities that the teaching staff can take advantage of. In addition, the platform encourages network of educational activity between the child and his/her school.

Dewi Rooslani Tojib et al. [12] develop and validate the business-to-employee portal user satisfaction (B2EPUS) measure. Experimental and confirmatory factor evaluation confirmed an 18-item, five-factor measure of B2E portal user satisfaction, namely Usefulness, Confidentiality, Ease of Use, Convenience of Access, and Portal Design as the dimensions of the construct. The presence of the second-order factor $x^{2}$ from the competing model evaluation, shows that B2EPUS concept corroborates the opinion that the user satisfaction concept should not be handled as a single construct as regularly proposed.
Advanced Encryption Standard (AES) algorithm was proposed in [4] to secure personal information provided by clients, its design principle is based on substitutionpermutation network. Contrasting to its precursor Data Encryption Standard (DES), in Advanced Encryption Standard (AES), Mail Response System was included as an additional feature to update members as regards recent changes in some information that possibly will interest user. An exclusive attribute has been added for posting reviews of blogging in AES, in addition, members can store information according to his/her preferred location and budget.

Diasy Seng, Ly Fie Sugianto and Carlo Wilkin [13] extends research knowledge about user satisfaction to mobile portals environment. Numerical findings in this study for mobile portal user satisfaction (MPUS) have established that, perceived usefulness (PU) and perceived value (PV) are not the same from that reported in the erstwhile literature and the new form, device specific (DS) and personalized interface (PI) are needed to capture attributes of user unique to mobile portals. A novel reliable and acceptable instrument mobile portal user satisfaction (iMPUS) was also developed to establish the mobile portals statistical validity and reliability. The connection with external validity is one of the inadequacies of this study. The broad view of the findings in this study was restricted from typical environments as a result of data collection was only from individuals who used mobile devices.

Leandro V. Silva et al. [14] proposed a software architecture that supports multiple methods to secure data aggregation. The software architecture makes possible the use of cloud computing in applications with stringent privacy and security requirements. In their work, two operations of the secure aggregation module were considered. One of this operation was based on Intel SGX (Software Guard eXtensions) technology, which does not insist on high computational costs, but requires processors that supports the software guard instruction sets. The second operation based on homomorphic encryption, which is completely programmed in software, but imposes high additional processing costs. Two use cases were focused on in their investigations: the estimation of consumers' monthly bill and the estimation of instant energy consumption in a region. Result shows that the aggregation strategy with Intel SGX proved to be much more efficient than homomorphic encryption.

While the number of people who use the Internet services is increasing, the importance of network media also increases. People are increasingly attracted by the fact that the Internet media has more freedom [15], as opposed to the conventional types of mass media: television and print media. Information aggregates comes packed with lots of content and widgetready areas and manages to display everything in a clear and readable manner. Quite a lot of studies have investigated different news aggregators, with a view to determine how they are designed and implemented. Though, some aggregators have made these practice to be profitable by charging for adverts on their aggregator pages or becoming an affiliate of the Internet shopping sites they syndicate data from. While developing this WordPress website, we have wonder how readers will find the site. If there is a plan to use the site as a business, we will also worry about how customers will find this web based aggregate information portal. In this research, we examined which sites and designs are integrating 
different social affordances that are important for supporting and fostering web users' interaction and engagement.

\section{WeB-BASED PoRTAL DESIGN AND DEVELOPMENT PROCESS}

There are various strides in the site configuration and advancement process. From social occasion beginning data, to the formation of the site, lastly, maintenance to stay updated. The procedure of web-based portal configuration and advancement are: (1) Information Gathering; (2) Planning; (3) Design; (4) Development; (5) Testing and Delivery; (6) Maintenance.

The information gathering is really the most essential stage, as it includes a strong comprehension of the group the Web entryway is made for. It includes a decent understanding what the business objectives and dreams are, and how the web can be used to accomplish the set objectives. The data accumulated from stage one is assembled to outline an arrangement for your site. It is at this planning stage the web architect chooses what innovations ought to be actualized. Components, for example, intelligent structures, ecommerce, streak, and so on are examined when arranging your site. In the design stage the look and feel of the Web entryway is resolved. Target group of onlookers is one of the key components to be contemplated. A site went for kids, for instance, will look vastly different than one implied for a monetary foundation. As a major aspect of the outline stage, it is additionally imperative to fuse components, for example, the organization logo to reinforce personality on the site. In this stage, correspondence between both the planner and client is urgent to guarantee that the last site will match needs and taste.

The development stage is the point where the site itself is made. At this stage, the greater part of the individual realistic components from the model are utilized to make the real, practical site. At the testing and delivery stage, the last subtle elements and test on the web entry is led. Similarity issues, guaranteeing that our site is streamlined to be seen appropriately in the latest program variants. The gateway is conveyed, the records setup and transfer remedied on the server. FTP (File Transfer Protocol) project is utilized to transfer the site documents to your server. Space name enlistment and Web facilitating administrations should likewise be possible. Web entryway can be made open, this is the official dispatch of the website, as it is presently perceptible to people in general. Lastly, the maintenance stage involves upgrading the data on your site, improving the usefulness of the site to dodge out of date quality.

Fig. 1 shows the flow process of the proposed web-based aggregate information portal.

\section{A. System Analysis of the Proposed Web Portal}

Since there is no current framework the data gathering for the web entryway was done utilizing:

- Structural examination;

- Structured structure;

- $\quad$ Sourcing for open-source arrangements;

- Building the Model destinations;

- Testing and Delivery of the site.

This undertaking was surrounded in a way that it covers all the essential areas that must be fused in the configuration. It is on RSS channels site aggregator and take information from a specific given site.

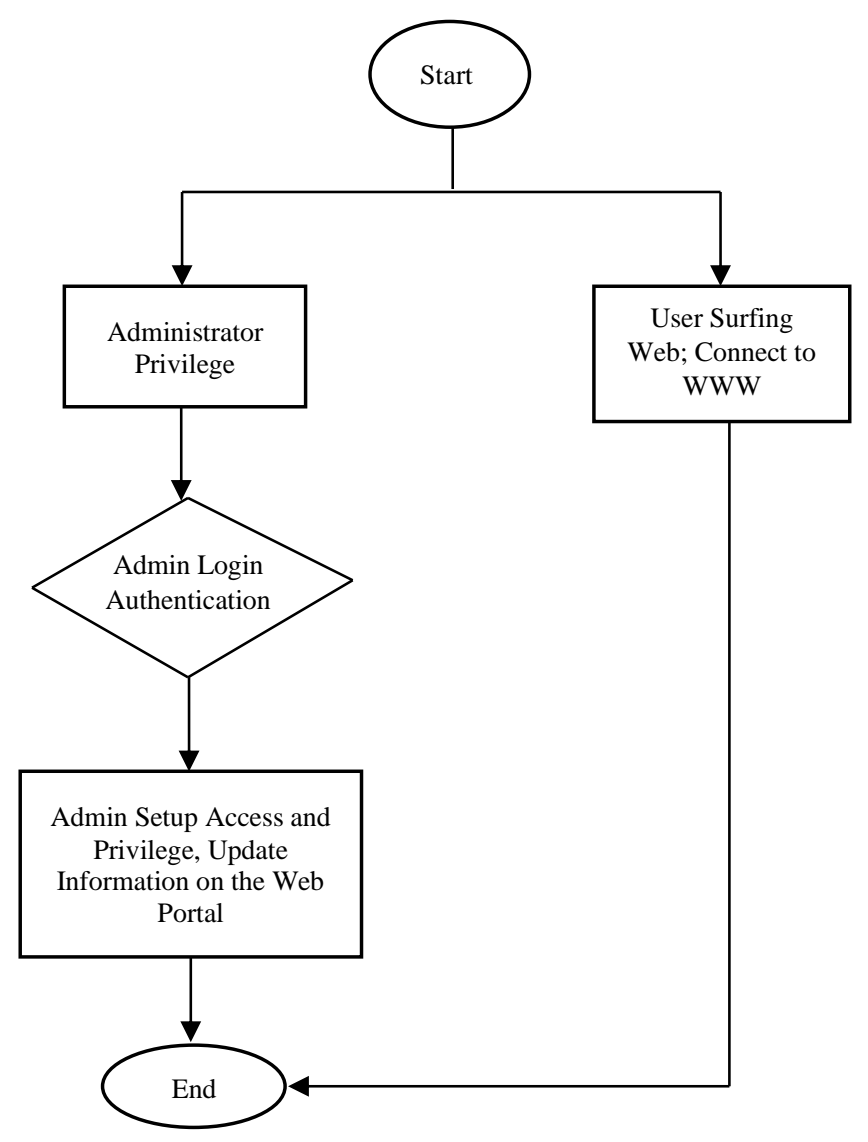

Fig. 1. Flow process of the proposed web based aggregator.

\section{B. Requirement of the Web-Based Aggregation System}

The undertaking was focused on sites that gives RSS channels, to make it less demanding for total data for clients surfing the site. The electronic total data gateway will serve people in general, and the proposed entry must be anything but difficult to-use, as it is a "beginning stage" for web surfing.

The necessities for Web based total data Portal were from an open source arrangements and are as per the following;

- XAMPP was introduced as shown in Fig. 2, which is windows construct and keeps running with respect to Apache, MYSQL and PHP.

- MYSQL database was created.

- WordPress version 4.2.1 aggregator module - a PHP framework was installed and used as a tool to collect syndicated material. WordPress requires stating of how often we want to update the site and the schedule of each time there is a new story from the feeds was chosen.

- Subsequently, we customized.

- Finally, we uploaded to server. 


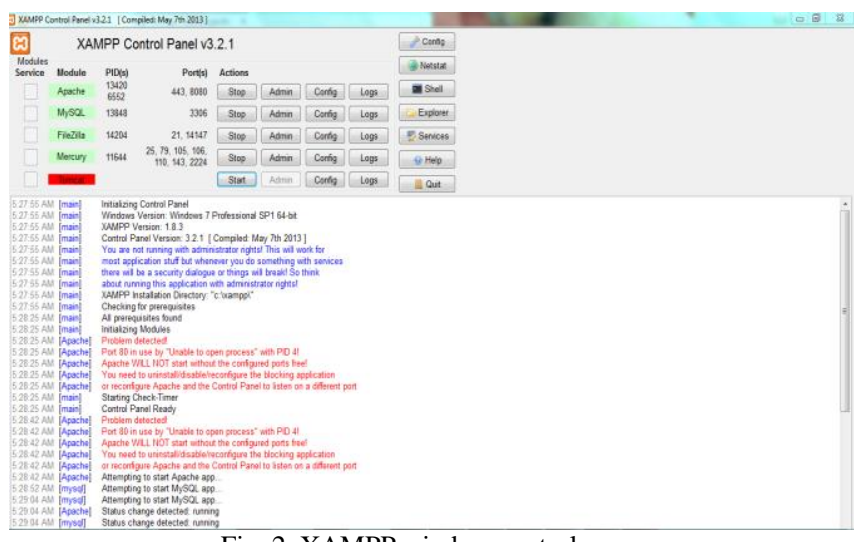

Fig. 2. XAMPP window control page.

\section{Basic Technology behind WordPress as Content Management System (CMS)}

Essentially, CMS is a system that deals with substance without expecting to do (much) coding. WordPress is a CMS. Looking at the WordPress theme first time (the actual files), it looks wild. There's a whole bunch of PHP, HTML, CSS, and sometimes JavaScript or jQuery, and that's not even getting into the other folders and files that make up WordPress core and all the plugins.

The step by step break down of WordPress technology are as follows:

\section{Entering content}

From utilizing any blogging stage and acquainted with a WYSIWYG ("what you see is what you get") post editorial manager. Those editors are simply favor shapes that spit the data we enter (title, substance, labels, and so on.) into a database.

\section{PHP in theme grabs the content}

The subject uses a bundle of PHP format labels to haul that substance pull out of the database. These arrive in an assortment of structures, yet they'll regularly look something like:

[php]
$<$ ?php
$<$ ?php
[/php]

the_title();

the_content();

?>

?>

\section{HTML in theme provides structure}

In subject, PHP is justifiably (as a rule? continuously? I think dependably yet can most likely be demonstrate wrong by some person) encompassed by HTML, which gives the page format structure. Once in a while those PHP labels will likewise produce some of their own HTML, so we may not generally see all the HTML right on the page when we take a gander at a topic document.

For instance, beneath rapped our title and substance into HTML headers and divs:

[html]

$<$ header class="entry-header" $>$

$<$ h1 class="entry-title" $><$ ?php the_title ()$; \quad$ ? $></$ h1 $>$

$</$ header $>$

$<$ div class="entry-content" $>$

$<$ ?php

the_content();

$</$ div $>$

[/html]

\section{CSS in theme styles the content}

In the event that we need the title to look unique in relation to the post body (ordinary). The CSS in the subject is the thing that gets that going. Programs have default CSS that will do some of this, yet ordinarily not with an eye to excellence so subjects normally indicate this in their CSS templates.

[css]

h1.entry-title

color:

orchid;

text-transform:

uppercase;

\}

[/css]

\section{JavaScript \& jQuery add interactivity}

The last layer is intelligence. There are some covers in the middle of CSS and jQuery here, in that occasionally they do a tiny bit of each other's' occupations, yet when all is said is done jQuery is utilized to add intuitiveness and fun impacts to a site, for example, light boxes on pictures, slideshows, extravagant drop downs, and so forth.

\section{Design Procedure}

A procedure model lets us know about how the information is handled and how the dataflow starting with one table then onto the next, to assemble the required data. This model comprises of the following:

- Information Flow Diagram;

- Data Flow Diagram.

\section{Information Flow Diagram}

An information flow diagram demonstrates the stream of data from its source to its destination through some medium. An information stream is spoken to by a line, with sharpened stones showings the course of stream.

\section{Data Flow Diagram}

The Information stream chart is the beginning stage for more point by point procedure graph called data flow diagram (DFD). A data flow diagram shown in Fig. 3 is a graphical representation of the stream of information between procedures.

\section{E. Database Design}

A Web gateway must be upheld by a vigorous and secure database which can store a lot of information. The structure of the database must have the capacity to bolster snappy recovery of information. This segment portrays the information model use in communicating with the information structures of the database supporting the electronic total data entry. An information model delineates the dataflow and legitimate interrelationship among diverse information components. The database design, WordPress MYSQL database and the text structure of the proposed system are shown in Fig. 4, 5, and 6, respectively. 


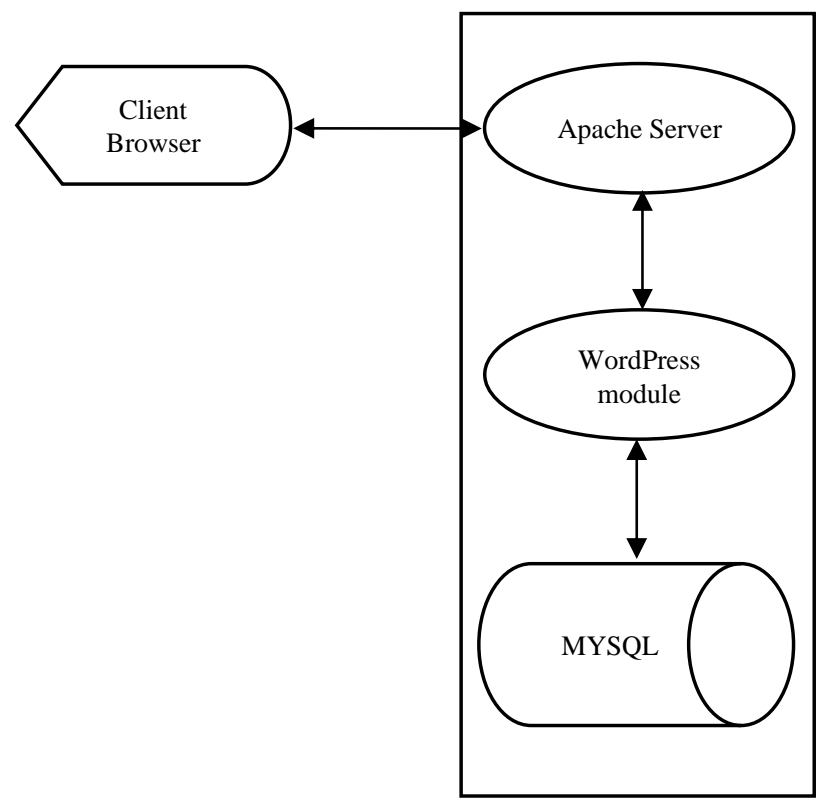

Fig. 3. Data flow diagram for the proposed system.

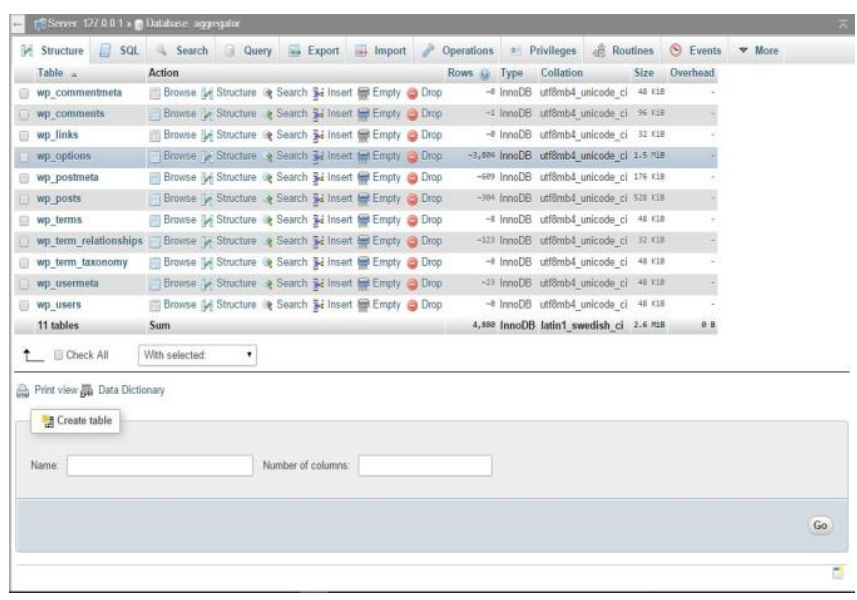

Fig. 4. Image of database design for the proposed system.

\begin{tabular}{|c|c|c|c|c|c|c|c|}
\hline \multicolumn{2}{|c|}{ 闰Browse } & \multirow{2}{*}{ 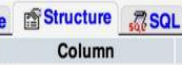 } & \multirow{2}{*}{$\frac{\text { Search }}{\text { Type }}$} & \multicolumn{3}{|c|}{ 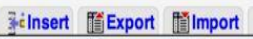 } & \multirow{2}{*}{$\frac{2 \text { Operations }}{\text { Default }}$} \\
\hline & $\#$ & & & Collation & Attributes & Null & \\
\hline 0 & 1 & $\underline{\mathbb{D}}$ & bigint(20) & & UNSIGNED & No & None \\
\hline 0 & 2 & post_author & bigint(20) & & UNSIGNED & No & 0 \\
\hline 0 & 3 & post_date & datetime & & & No & $0000-00-0000: 00: 00$ \\
\hline$\square$ & 4 & post_date_gmt & datetime & & & No & $0000-00-0000: 00: 00$ \\
\hline 0 & 5 & post_content & longtext & utt8_general_ci & & No & None \\
\hline 0 & 6 & post_title & text & utfi_general_ci & & № & None \\
\hline 0 & 7 & post_excerpt & text & utt8_general_ci & & No & None \\
\hline$\square$ & 8 & post_status & varchar(20) & utti__general_ci & & No & publish \\
\hline 0 & 9 & comment_status & varchar(20) & utt8_general_ci & & No & open \\
\hline$\square$ & 10 & ping_status & varchar(20) & utt8_general_ci & & No & open \\
\hline$\square$ & 11 & post_password & $\operatorname{varchar}(20)$ & utt8_general_ci & & No & \\
\hline 0 & 12 & post_name & varchar(200) & utf8_general_ci & & No & \\
\hline 0 & 13 & to_ping & text & utt8_general_ci & & No & None \\
\hline 0 & 14 & pinged & text & utt8_general_ci & & No & None \\
\hline
\end{tabular}

Fig. 5. WordPress MYSQL database for the proposed system.

\section{F. Web Base Development}

The Web is built on the Hypertext Transfer Protocol (HTTP). HTTP is a client/server request/reply protocol that is stateless. That is, the protocol does not make any association between one transaction and another, e.g.: time since the last transaction, type or client involved in the last concerned, each transaction is a discrete event.

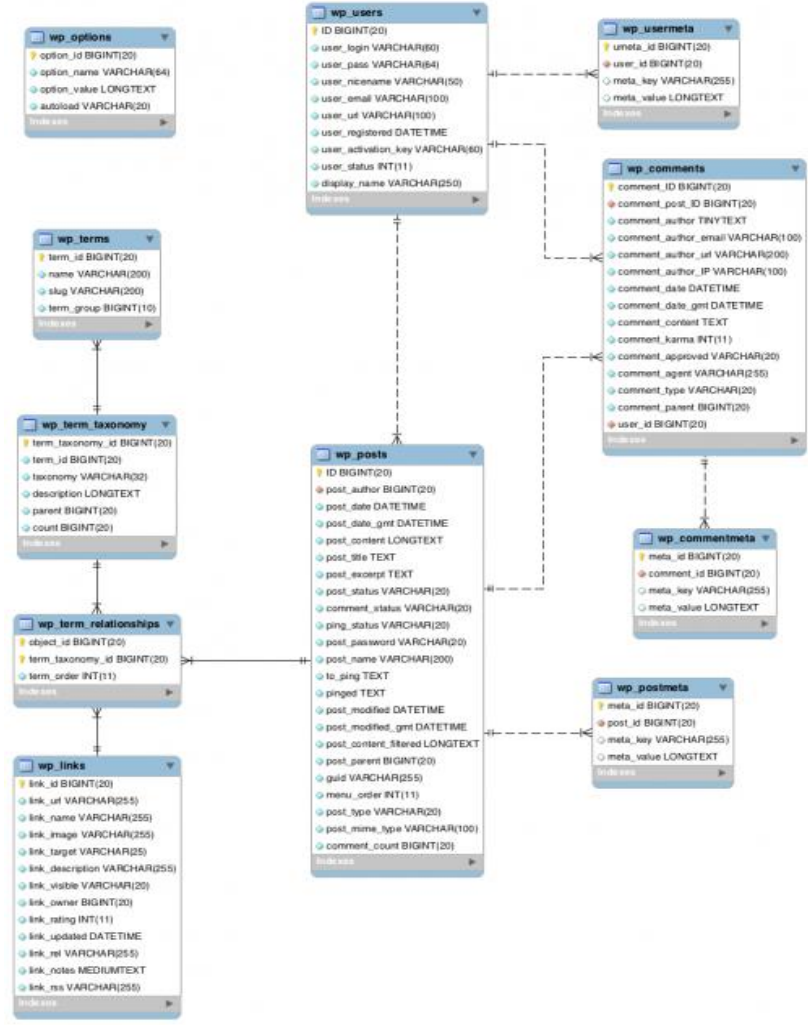

Fig. 6. Text structure for the proposed system.

\section{G. MYSQL Database Connectivity Using XAMPSERVER}

In this research, MySQL is used as the backend database. MySQL is an open source database management system. The features of MySQL are given below:

- MySQL is a relational database management system. A relational database stores information in different tables, rather than in one giant table. These tables can be referenced to each other, to access and maintain data easily.

- MySQL is open source database system. The database software can be used and modify by anyone according to their needs.

- It is fast, reliable, and easy to use. To improve the performance, MySQL is multithreaded database engine. A multithreaded application performs many tasks at the same time as if multiple instances of that application were running simultaneously.

In being multithreaded MySQL has many advantages. A separate thread handles each incoming connection with an extra threat that is always running to manage the connections. Multiple clients can perform read operations simultaneously, but while writing, only hold up another client that needs access to the data being updated. Even though the threads share the same process space, they execute individually and because of this separation, multiprocessor machines can spread the thread across many CPUs as long as the host operating system supports multiple CPUs. Multithreading is the key feature to support MySQL's performance design goals. It is the core feature around which MySQL is built.

PHP was used because its server side language is capable to generate dynamic web pages which allow its users to interact with the owner or with the other users. MySQL is database management program that is compatible with all operating system therefore they are cross-platform compatible. Everything is $100 \%$ remotely configurable. They have fast and easy development environment. They have 
multi-lingual support so widely used in all parts of the world. They can use in any scale of development and its examples are Facebook and Wikipedia as both websites are made up of PHP MySQL development. MySQL is an excellent relational database management system (RDBMS), even we can use flat file for database integration which allows easy changes in your database.

\section{H. System Requirements}

The requirement for the successful implementation of this research can be categorized into two namely - Hardware and Software requirement.

\section{Hardware Requirement}

The following computer and systems hardware is required for implementation of this research:

- A computer system which has Windows XP, Windows Vista, Windows 7, Windows 8, Windows 10, Mac, Linus or Unix.

- 10 Gigabyte and above hard disk.

- 256 Megabyte RAM and above memory.

- $\quad 1.0 \mathrm{GHz}$ processor and above.

- Phones, Pad's and any Gadget that surf's Internet.

\section{Software Requirement}

The following software are required for implementation of the research:

- XAMPP Server.

- MySQL.

- WORDPRESS version 4.2.1.

- RSS feeds: Really Simple Syndication (RSS) is a widely used technology for transmitting information across the Internet and intranets.

- Browser - Internet Explorer, Firefox, Opera, Safari, Google Chrome and so on.

\section{SYSTEM IMPLEMENTATION}

\section{A. Kasere Multiconcept Description}

Kaseremulticoncept is a domain name of the Web portal that provides a single point of access to application and information. The end user will see it as a web site with pages that are arranged by tabs as form of navigation. Each page contains sub-pages of individual windows that will display from static HTML content to complete complex web services. This gives users access to different information and tools in a single place. It provides the uniform resource location (URL) for user's access, serving as a consistent, unified web-based interface.

The creation and management of kaseremulticoncept portal contains these phases:

- Architecture: the basic configuration of Web portal was determined.

- Development: Pages was created for aggregation of information on the web.

- Staging: the web portal was deployed to a staging environment, assembled, and tested with Administration console.

\section{Administrator Profile}

Fig. 7 shows a login interfaces for the administrator and authentication using PHP script language to communicate with MySQL. A unique ID is given to the administrator and a password is being generated. The unique ID is used to login to their profile to regulate and update the web based aggregate information portal page as shown in Fig. 8.

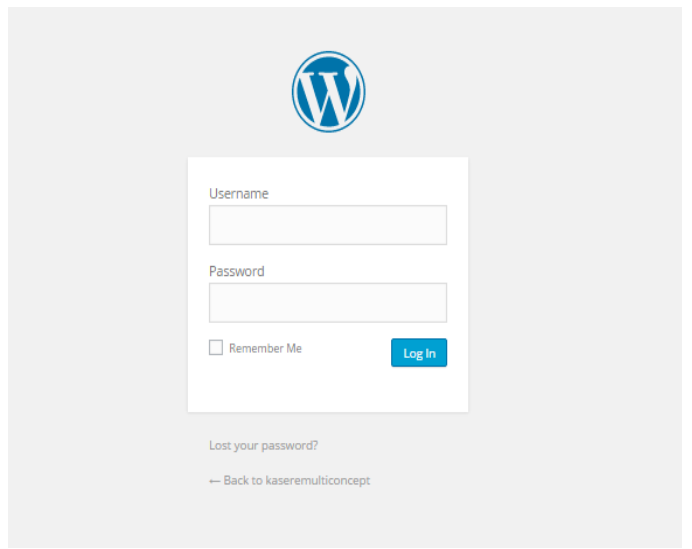

Fig. 7. Administrator login interface.

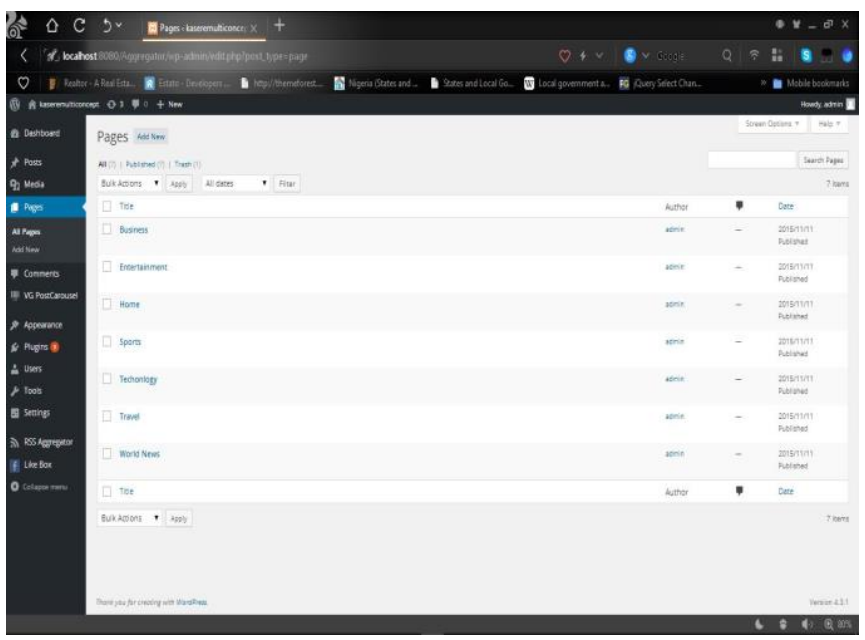

Fig. 8. Web based aggregate information portal page.

The content of kaseremulticoncept web based aggregate information portal pages in Fig. 8, collects information from different platform sources into a single user interface, providing users with relevant information are as follows:

\section{Home Page}

This displays the default webpage that loads when kaseremulticoncept Web based aggregate information portal is visited. All the pages are shown in a web browser when the application starts up for the end user as shown in Fig. 9.

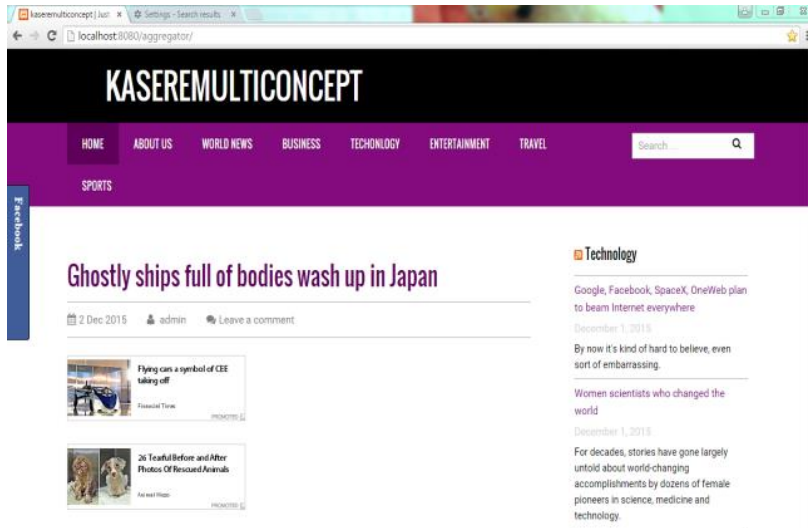

Fig. 9. Web based aggregate information home page.

\section{World News}


The world news webpage in Fig. 10, is organized hierarchically to showcase aggregated international news on Web for end user's consumption, to offer and provide the information that users need.

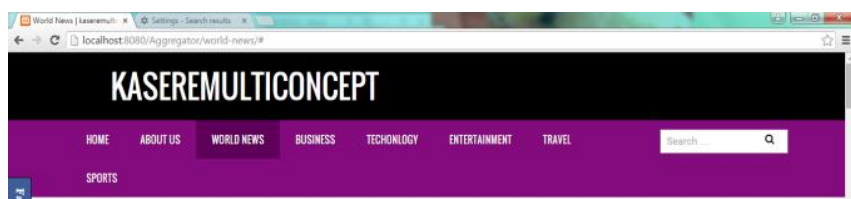

\section{World News}

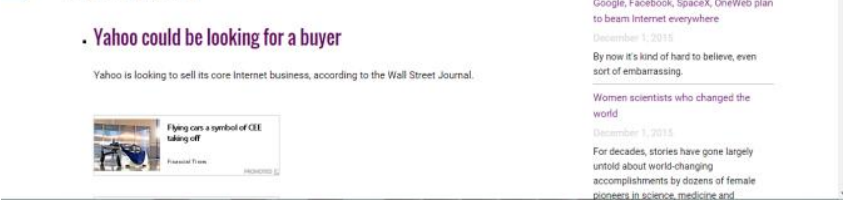

Fig. 10. World news webpage.

\section{Business}

The business webpage shown in Fig. 11, is organized hierarchically to showcase aggregated international business news on stock markets, financial \& earnings, Web for users' consumption, to offer and provide the information on currencies futures.

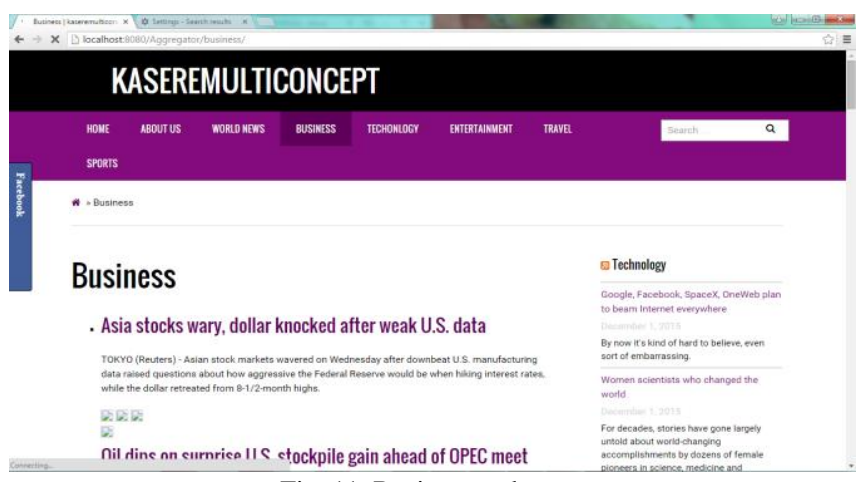

Fig. 11. Business webpage.

\section{Technology}

The technology webpage shown in Fig. 12, is organized hierarchically to showcase aggregated international information relating to new gadgets and technology news on Web for users' consumption, to offer and provide the information that users need.

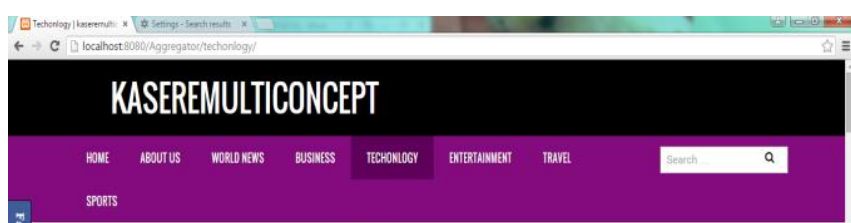

Techonlogy

China behind 'massive' cyber-attack on Australian government: $A B C$

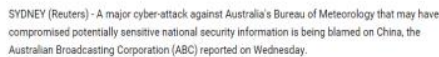
20.6.

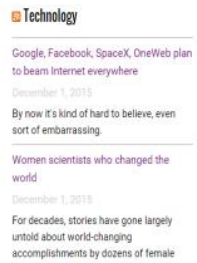

Fig. 12. Technology webpage.

\section{Entertainment}

The entertainment webpage shown in Fig. 13, is organized hierarchically to showcase aggregated International Entertainment industry news on Web for user's consumption, to offer and provide the information that users need.

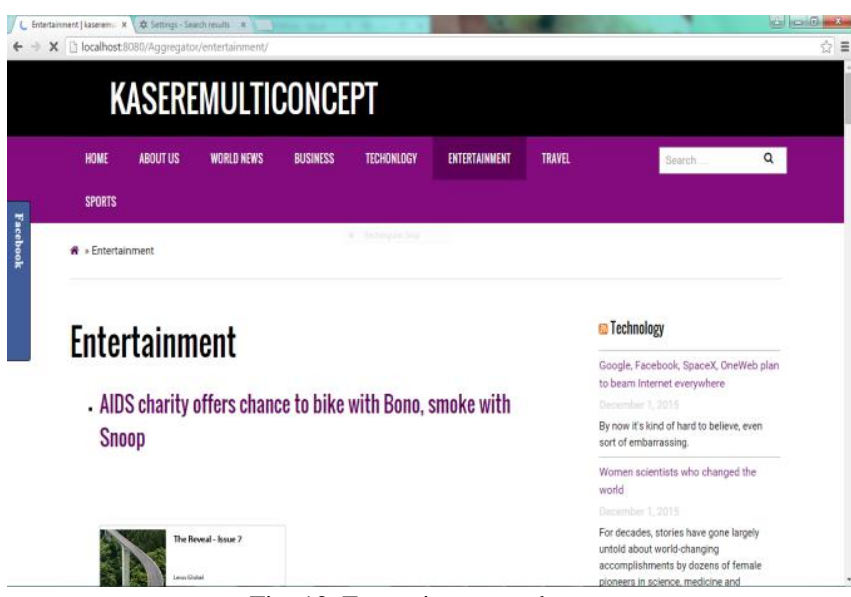

Fig. 13. Entertainment webpage.

\section{Travel}

This webpage is organized hierarchically to showcase aggregated International Travel news on Web for user's consumption, to offer and provide the information that users need.

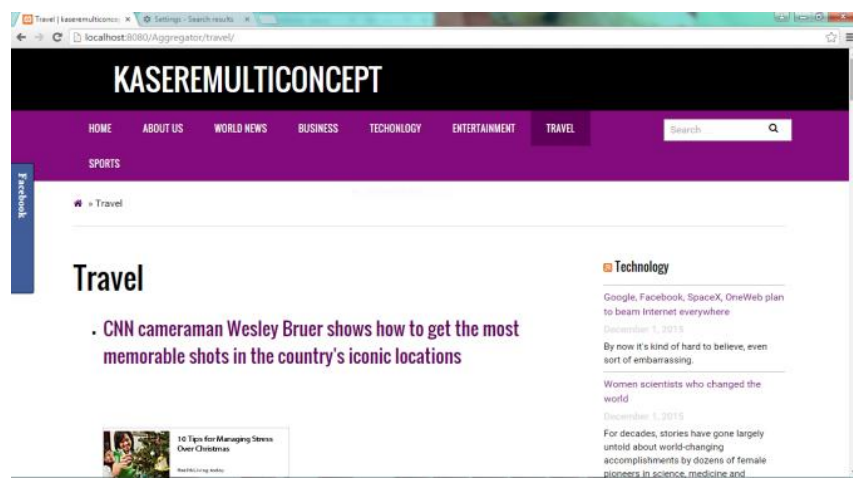

Fig. 14. Travel webpage.

\section{Sport}

This webpage is organized hierarchically to showcase aggregated International sports news on Web for user's consumption, to offer and provide the information that users need on sports.

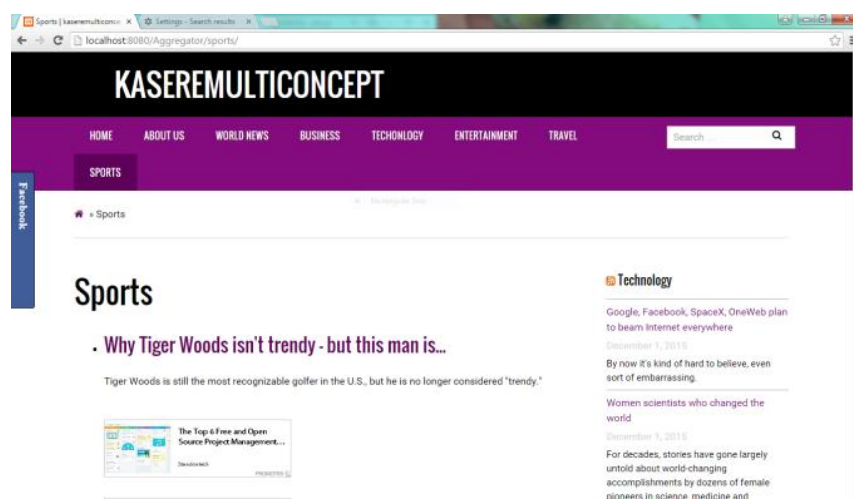

Fig. 15. Sport webpage. 


\section{CONCLUSION}

In this research, we have designed an easy-to-use portal for aggregate information in some particular area of interest, which has been captured on the Web pages. The following have been achieved:

- Build an Aggregate information websites that collect and post syndicated material from around the Web (selected websites), including news, specialized reports in business or world news, or the latest bargains and deals in Internet travel.

- Enhanced this news aggregate information to provide a single source for each headline and do not cluster together news articles from different sources reporting on the same story. This results in a simple laundry list of different top stories, each with a unique single source for content.

- An interactive portal which is a gateway to social network sites Facebook, and more popular search engines: Search.

- Administrator Authentication very secure.

- Provide Users whatever added information aggregate they needs based on the selected websites and web page.

\section{LIMITATIONS}

This research has presented different news aggregators, with a view to determining how these are designed and implemented. Each of these true aggregators has some social affordances built in, primarily message posting. However, user messages or blogs that are included are often not displayed in relation to the news stories they are commenting on. Rather, they are listed as a separate set of content, thereby disconnecting them from the news or issue they may relate to.

From browsers to screen resolutions, to user interactions, we seem to constantly be struggling to find some way of thinking outside the tiny little box of "best practice" which we are constrained by. The most important part of any design is the message: good design sends the same message to everyone. It should leave no room for interpretation. Digging down into specifics, we can look at some key areas where limitations can greatly improve design. The first of these areas is color, limiting your color palette is extremely important. Reducing the number of colors, we use in our design will make the piece feel consistent. Basically, everything will look like it goes together.

Typography works in a similar way to color, if not on a more extreme scale. Bar a few exceptions, over-using many fonts within a single design can become confusing and even difficult to read. The human eye likes to settle into patterns of recognition, introducing brand new types of type (pun intended) can greatly hinder this and interrupt the flow reading. Restricting the use of typography, once again, contributes to a better and a stronger overall design.

\section{REFERENCES}

[1] A. Z. Idris and N. A. Yahaya, "Design and Implementation of an Aggregation-based Tourism Web Information System," vol. 9, no. 12, pp. 143-148, 2009.

[2] D. Jalal, M. M. Al-debei, "Portals and Task Innovation : A Theoretical Framework Founded on Business Intelligence Thinking," pp. 1-20.

[3] Ramona, W. (2001) portals: - The all in one web supersites features, functions, definitions, taxonomy. Available from: http://www.sapdesignguild.org/edition3/portal definition.... Access October 2011

[4] D. Hasabe, N. Mane, R. Sanap, A. Gursale, "Web Portal for providing various services," vol. 2, no. 3, pp. 2014-2016, 2015.

[5] I. Afrianto, A. Heryandi, and L. Warlina, "Online Journal Aggregator System Design Using User Centered Design ( UCD ) Online Journal Aggregator System Design Using User Centered Design ( UCD ) Approach," no. January, 2019.

[6] Boye, J. Are all portals the same? Internet related technologies, 1999 Available from: http://tech.irt.org/articles/js147/ Access October 2011

[7] Melzer, C. Enterprise information portals. South African Journal of information Management, 1(2/3):1, $1999 . \quad$ url: http://general.rau.ac.za/inosci/raujournal/vol1.nr2.01_09_99/default.as p?to=webs. Access October 2011.

[8] Webopedia portals available from http://ww.wikianswer/Q/what_is_webportal accessed October 2011.

[9] Madam Sheina, et al. Ovum ltd enterprise portals: New strategies for information delivery ovum reports (Book), 2000.

[10] G. Paliouras, A. Mouzakidis, and C. Skourlas, "PNS : A Personalized News Aggregator on the Web PNS : A personalized news aggregator on the Web," no. June 2014

[11] F. Ali, A. Hayek, M. M. Khelaif, Z. Ali, A. Shaikh, H. K. Alshammari, M. Alshabanah, D. Alrajhi, M. K. Alsmadi, and I. Almarashdeh, "Developing and Implementing a Web-Based educational platform for Children with Special Needs," vol. 7, no. 2, pp. 189-200, 2020.

[12] D. R. Tojib, L. Sugianto, and S. Sendjaya, "User satisfaction with business-to-employee portals: conceptualization and scale development," pp. 649-667, 2008.

[13] D. Seng, L. F. Sugianto and C. Wilkin, "Measuring Mobile Portal User Satisfaction," pp. 1-12, 2016.

[14] L. V Silva, P. Barbosa, R. Marinho, and A. Brito, "Security and privacy aware data aggregation on cloud computing," 2018.

[15] V. Andrunyk and A. Vasevych, Development of Information System for Aggregation and Ranking of News Taking into Account the User Needs, 2020 .

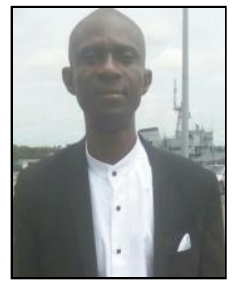

Donatus I. Bayem received his B.Eng. degree in Electrical/Electronics Engineering from Ambrose Alli University, Ekpoma, Nigeria in 1998, and MIT degree from University of Lagos, Nigeria, in 2016

In 2000, he worked at NEET Panasonic Ltd, Port Harcourt as Head, Service Center Engineer and in 2002 worked at COSCHARIS TECHNOLOGIES Ltd Port Harcourt as Sales Engineer. In 2003, he worked with ACTI-TECH Ltd, Abuja-Nigeria as Resident Engineer. In May 2005, he worked at Halogen Security Company Ltd, as Senior Information Technologies Analyst to set up Technologies department and in 2018, he worked at Real Strikers Security Services Ltd as Head, ICT \& Protocol Services. He was involved in severally developing and executing turnkey projects for clients comprising, multinational companies, Government parastatals, Banks, Schools, Telcos and oil industries. Currently, he is a Lecturer in the Department Electrical Engineering, Nigeria Maritime University, Okerenkoko, Delta State, Nigeria.

He has certificate in Project Management from Beni American University and Diploma certificate from Axis communications Academy, South Africa He has Cisco Certified Network Associate (CCNA) in 2011 and Microsoft Certified Technical Specialist (MCTS) in 2011

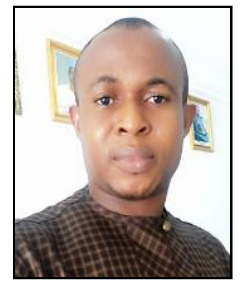

Henry O. Osuagwu obtained his B.Eng. Electronic Engineering and M.Eng. Communication Engineering from University of Nigeria, Nsukka, Nigeria in 2011 and 2016 respectively. He is currently a Ph.D. student in the Department of Electronic Engineering, University of Nigeria, Nsukka, Nigeria. He is a member of Nigerian Society of Engineers (NSE) and a registered COREN engineer. He is a Lecturer in the Department of Electrical Engineering, Nigeria Maritime University, Okerenkoko, Delta State, Nigeria. His research interests include Wireless Communication, Radio Resource Management, Network Security, and Cognitive Radio in Wireless Sensor Networks. 


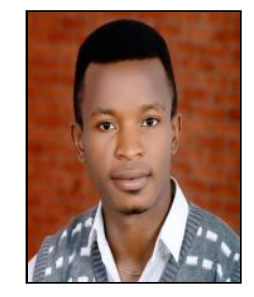

Chimezie F. Ugwu holds a Bachelor of Science degree in Computer Science and Statistics from the University of Nigeria Nsukka. Master of Science Degree in Computer Science at the University of Nigeria, Nsukka and he is currently pursuing his $\mathrm{Ph} . \mathrm{D}$. at Ebonyi State University, Nigeria. He is a Lecturer in the Department of Computer Science, Institute of Management and Technology, IMT

Enugu, Nigeria. His area of research interest includes: Ethical hacking and Network Security. 